\title{
EL VOTO \\ EXTRATERRITORIAL Y \\ LA CÁMARA DE DIPUTADOS DE \\ MÉXICO (1994-2005) \\ JOSÉ FRANCISCO PARRA*
}

RESUMEN. La interacción partidista sobre el voto de los mexicanos en el exterior puede ser analizada mediante la teoría de juegos. La Cámara de Diputados de México ha sido un actor importante en el ciclo de reformas electorales y, al mismo tiempo, el foro donde se ha debatido la incorporación de los ciudadanos mexicanos residentes en el extranjero en el proceso electoral. Las posturas de la élite partidista mexicana responden directamente a la evaluación utilitarista de costos y beneficios, y no a una causa ideológica o normativa.

Palabras ClaVe. México, emigración, élite parlamentaria, teoría de juegos, voto en el extranjero.

Abstract. Partisan interaction over the vote of Mexicans overseas can be analyzed in terms of game theory. Congressional members of Mexico Parliament have been important actors in the cycle of electoral reform and, at the same time, a forum for debating the incorporation of Mexicans resident overseas into the electoral process. The diverse positions taken by Mexico's political class respond directly to a utilitarian calculation of costs and benefits rather than a normative or ideological positioning. KEYwORDS. Mexico, migration, parliamentary elite, game theory, extraterritorial.

* Investigador del Centro de Estudios de México en la Unión Europea, Instituto Universitario de Investigación Ortega y Gasset, Madrid. 

transformación de sus instituciones políticas. Este cambio incremental se ha reflejado en la política del Estado hacia los ciudadanos transmigrantes mexicanos (Santamaría, 1994, 2001; Martínez Saldaña, 1993; Calderón, 1997, 2003; Calderón y Martínez, 2003; Fitzgerald, 2000). Es decir, la transición a la democracia, en México, ha sido un proceso intenso y difícil donde los mexicanos normalizan las prácticas democráticas, de tal manera que los cambios han sido producto de una combinación de factores institucionales: 1) la estructura de incentivos de la élite política; 2) los factores socioeconómicos así como estructurales; y 3) el contexto nacional e internacional. ${ }^{1}$ Los efectos más importantes del cambio en las instituciones, principalmente en las leyes electorales, han sido la implementación de nuevas reglas del juego que regulan la competencia política y el aumento en la participación electoral de segmentos de la sociedad antes inactivos. Ese proceso no había incluido a los ciudadanos emigrantes en el exterior, al menos antes de la reforma de junio de 2005, que, finalmente, ha reglamentado el voto postal para emigrantes en las elecciones presidenciales de $2006{ }^{2}$

Otra de las consecuencias institucionales de la espiral de reformas electorales ha sido un protagonismo inusitado del Poder Legislativo, de manera principal la Cámara de Diputados. Históricamente, los arreglos institucionales del sistema político, tanto de iure como de facto (Cosío Villegas, 1975; Carpizo, 1991), le permitieron al presidencialismo mexicano ejercer un predominio sobre los otros dos

\footnotetext{
${ }^{1}$ Para algunos autores como Becerra, Salazar y Woldenberg (2000), la transición mexicana proviene "desde abajo", de la periferia al centro, con la intervención de una pluralidad de partidos políticos. Estos autores señalan que los síntomas de la democracia aparecieron, primero, a escala estatal y local antes que nacional: gobiernos divididos, poder compartido, triunfos electorales regionales, desahogo jurisdiccional de las controversias, alternancia. Al mismo tiempo, dicen Becerra et al. (2000), uno de los grandes fenómenos democráticos ha sido la participación de los ciudadanos, es decir, la transición no sólo transformó las reglas electorales en un escenario de participación electoral limpia y transparente, para partidos así como ciudadanos, sino que fue impulsora de una auténtica ciudadanía política.

${ }^{2}$ Es importante aclarar que el derecho al voto de los mexicanos en el extranjero, al fin, ha sido reglamentado en junio de 2005, aunque, originalmente, fue aprobado por el Congreso mexicano en la reforma electoral de 1996. En este impasse, que va de 1996 a 2005, se asumieron diversas posturas al respecto, por ejemplo, para autores como Woldenberg (2002) no era un problema de discriminación política; en tanto que para otros no era un asunto sujeto a «discusión» (Szekely, 2003), lo que sí estaba a debate era su operatividad. A nuestro entender - y en términos prácticos- lo que existía era una exclusión política de los emigrantes mexicanos, ya que se trataba de un derecho político real pero que, simple y sencillamente, no se podía ejercer. En términos de la teoría de la justicia, las posturas más comprehensivas se hallaban en Santamaría (1994) y Calderón y Martínez (2002), que exigían que el Estado mexicano se reconociera como país de emigrantes, para, a continuación, dar paso a una cultura política en la que los derechos de los emigrantes fueran parte de la normalidad democrática.
} 
poderes de la Unión. ${ }^{3}$ Los efectos mecánicos, de las reformas políticas y electorales, se reflejan en resultados electivos con múltiples ganadores y una composición multipartidista de la representación parlamentaria, misma que abre el camino a una relación diferente entre los distintos poderes. Estos cambios son relevantes por las facultades que tiene, la Cámara de Diputados, para reformar el marco legal de la competencia política y electoral, en el que también se encuentra la reglamentación del voto en el extranjero.

En este contexto de cambio institucional, un avance importante ha sido el establecimiento de reglas del juego auto impuestas, con resultados electorales confiables y respetados por los distintos actores políticos, se trata de un proceso de certidumbre electoral que se refleja en una atmósfera de confianza ciudadana sobre la utilidad del voto y las elecciones (Pérez y Martínez, 2000; Becerra, Salazar y Woldenberg, 2000). Podemos afirmar que, la transición mexicana a la democracia, ha utilizado las reformas electorales como el instrumento para el cambio institucional y la Cámara de Diputados ha sido el escenario de su convenio así como de su aprobación (Elizondo y Nacif, 2002, Woldenberg, 2002). Por lo mismo, la negociación del voto de los ciudadanos mexicanos en el extranjero, invariablemente, también ha pasado por ahí. ${ }^{4}$

En este sentido, el objetivo de este artículo consiste en explicar cómo la interacción partidista en el ejercicio legislativo, entre 1994 y 2003, dejó fuera del proceso de toma de decisiones a los mexicanos en el extranjero. A pesar de una percepción aparentemente favorable de la élite parlamentaria respecto a la emigración, al final, los cálculos estratégicos (costo-beneficio) y la incertidumbre de sus resultados se impusieron a la creación de una ciudadanía democrática para los migrantes. Para cumplir con su objetivo principal, este trabajo se organiza en dos apartados. El primero revisa la relación política histórica del Estado con la emigración mexicana, la cual, en 2000, ascendía a más de 10 millones de ciudadanos, 99\% de los cuales radicaban en Estados Unidos. Lo anterior es un elemento imprescindible para analizar la estructura de incentivos de las élites políticas y partidistas, que fijan su posición, respecto al voto extraterritorial, sobre la base de una evaluación

\footnotetext{
${ }^{3}$ El sistema político mexicano siempre fue un espécimen raro de clasificar, como se ilustra en el trabajo pionero de Duverger (1986), o bien Silva-Herzog Márquez (1999). En términos generales, el modus operandi del presidencialismo mexicano se caracterizaba por dos condiciones básicas: 1) por ser una coalición que se desenvolvía en torno al Partido Revolucionario Institucional (PRI) — como una suma de intereses donde coincidían empresarios, campesinos, estudiantes, revolucionarios, partidarios del nacionalismo, capitalistas - que se destacó por una inusitada estabilidad, teniendo a la figura del presidente como el máximo responsable en la escala jerárquica, y 2) un sistema electoral no competitivo que mantenía el status quo de la coalición gobernante, es decir, ausencia de partidos capaces de competir e inexistencia de reglas electorales abiertas.

${ }^{4}$ Martínez Saldaña (2002) señala que incluir el derecho al voto en el extranjero, en el debate legislativo, ha sido un avance importante en la adecuación de la ciudadanía en México, a raíz de que los emigrantes se convierten en actores político-electorales visibles.
} 
de costos y beneficios electorales. La hipótesis de este trabajo es que la reglamentación del voto extraterritorial depende de la percepción político-electoral de las élites políticas y partidistas en lo referente a la emigración mexicana transnacional: inocua, arriesgada o amenazante. La segunda parte analiza la interacción partidista de la Cámara de Diputados, en México, durante los últimos tres periodos legislativos (1994-2003). En este sentido, el argumento principal es que las reformas electorales han sido importantes para el avance democrático del país, pero, al mismo tiempo, fueron insuficientes en términos democráticos, al dejar fuera el voto extraterritorial. El instrumento de análisis de este apartado es la teoría de juegos, ${ }^{5}$ que nos permite explicar la interacción entre actores que, en 1996, propiciaron cambios en las instituciones político-electorales. Al mismo tiempo, se utilizan estos esquemas para explicar el intento fallido de reforma en 1999, justo antes de las elecciones presidenciales de 2000. Finalmente, el artículo cierra con un análisis de la reforma electoral que permitirá, por primera vez, participar electoralmente a los emigrantes mexicanos en el extranjero en las elecciones presidenciales de 2006. Sin duda, haber reglamentado el voto postal es un paso importante, pero, lamentablemente, no corresponde a la realidad de la emigración transnacional mexicana.

\section{LA DIMENSIÓN TRANSNACIONAL DE LA POLÍTICA EMIGRANTE MEXICANA}

De manera tradicional, la emigración mexicana a Estados Unidos había sido estudiada por distintas disciplinas de las ciencias sociales, principalmente destacaban la antropología y la sociología. Así queda constatado en el trabajo pionero de Gamio (1930, 2002) y en los trabajos posteriores de Bustamante (1983), Bustamante y Cornelius (1989) así como Bustamante, Delaunay y Santibáñez (1997), amén de un sinfín de investigaciones serias y detalladas que dan cuenta de la experiencia migratoria mexicana hacia el norte del continente americano. Sin embargo, estos trabajos se distinguieron por describir, analizar y predecir las características junto con el comportamiento de la emigración mexicana en aspectos económicos, demográficos, sociales y culturales. Por su parte, la esfera de la política, de algún modo, estuvo apartada del análisis, ya que los especialistas daban por hecho que los emigrantes terminarían, tarde o temprano, en asimilados al sistema político

\footnotetext{
${ }^{5}$ No es intención de este artículo discutir sobre la pertinencia metodológica de la teoría de juegos, pero su aporte al análisis del cambio o inercia institucional han sido importantes para la ciencia política. La teoría de juegos no es la elección racional de un positivismo ortodoxo, al contrario, la construcción de esquemas de análisis son flexibles e incluyen un sinnúmero de variables explicativas que nos permiten encontrar resultados más apegados a la realidad. Por sólo citar un ejemplo, de la utilización de la teoría de juegos desde posturas comprensivas, mencionaremos la obra de Przeworski (1987, 1988, 1995).
} 
estadounidense, al tiempo que rara vez se contemplaba el aspecto político de la emigración y su vínculo con las instituciones políticas mexicanas. ${ }^{6}$

Pero, en la década de los noventa, y sobre todo a raíz del proceso electoral de 1988 — hito importante en la transición mexicana-, los estudios del aspecto político de la emigración mexicana a Estados Unidos empezaron a surgir de la mano de investigadores como Santamaría (1994, 2001), Martínez Saldaña (1993, 2002), Calderón $(1997,2002,2003)$ y otros más coyunturales, como Dresser (1995). En estos trabajos, los emigrantes en el exterior -y su incidencia en las instituciones políticas del Estado mexicano- se convirtieron en la variable de análisis, es decir, el estudio de la emigración transnacional mexicana como fenómeno politológico. Sin embargo, es importante reconocer que examinar el perfil político de la emigración mexicana fue posible gracias al desarrollo teórico del transnacionalismo, enfoque que permitió analizar el fenómeno migratorio como una cadena donde los principales eslabones son la comunidad de origen y la sociedad de destino, en la que destacan los siguientes parámetros: a) la intensidad de los flujos migratorios; b) la consistencia en el tiempo así como en el espacio; y c) el proceso de «ida y vuelta», entre comunidad de destino y de origen (Portes, Guarnizo y Landolt, 1999).

Lo anterior se puede constatar por varias razones. Primero, la migración mexicana es un fenómeno transnacional porque tiene un largo camino recorrido a través de los años, éste se remonta a 1848 y los primeros emigrantes mexicanos que «los cruzó la guerra» (1846-1848). ${ }^{7}$ En segundo lugar, la permanencia en el tiempo ha sido acompañada por un flujo nutrido y continuo de personas que, en los 150 años de experiencia migratoria, han cruzado la frontera para internarse en Estados Unidos. En tercero, lo transnacional se justifica por el intercambio permanente entre los lugares de origen y los grupos de emigrantes en el extranjero, proceso necesariamente de «ida y vuelta». Precisamente, este último ha sido uno de los fenómenos mejor reseñados de la experiencia migratoria internacional, a nadie deja de sorprender el grado de institucionalización transnacional de los mexicanos en el exterior, sobre todo cuando hablamos del envío de remesas a México, las cuales eran del orden de 12 mil millones de dólares en 2003 (Pew Hispanic, 2004). Asimismo, si nos referimos a la participación en programas de desarrollo comunitario transnacional entre lugares de origen y destino (González, 1995, y Moctezuma, 2000), o al mantenimiento de las principales tradiciones

\footnotetext{
${ }^{6}$ Para un estudio sobre las distintas corrientes teóricas que explican la asimilación de emigrantes mexicanos a Estados Unidos, desde un punto de vista politológico, se puede consultar el trabajo de Parra (2000).

7 Existe una bibliografía numerosa sobre la experiencia mexicana durante la guerra con Estados Unidos y las consecuencias que ésta tuvo para los emigrantes mexicanos. Para una revisión general se recomienda el trabajo de Meyer y Vázquez (1994). Para estudios más específicos, véase Acuña (1986), Montejano (1991) y Gutiérrez (1995).
} 
culturales, como el idioma y fiestas religiosas. Igualmente, por su asistencia en distintos momentos cruciales de la vida política del país, como la guerra contra Francia (1862-1867) (Castillo y Ríos, 1989, y Griswold, 1979), la revolución mexicana (1910-1920) (Raat, 1993, y Gómez-Quiñones, 1973) así como, más recientemente, en la transición democrática iniciada en el último cuarto del siglo xx (Santamaría, 2001; Calderón y Martínez, 2002; y Dresser, 1995).

A estas prácticas ejercidas por ciudadanos o grupos de mexicanos transmigrantes hay que sumarle el comportamiento del Estado mexicano, que ha contribuido a remarcar el aspecto transnacional de la emigración con políticas públicas como el Programa Paisano, el Programa Solidaridad Internacional entre Mexicanos (Fitzgerald, 2000), o la ley de «no pérdida de la nacionalidad mexicana» (Becerra Ramírez, 2000; Santamaría, 2000). Es decir, el Estado y el gobierno mexicanos se han adaptado al contexto internacional de la emigración transnacional con la finalidad de mantener el control sobre un amplio segmento de sus ciudadanos emigrantes. Sin embargo, este interés ha resultado ambiguo y contradictorio ya que, en ocasiones, ha sido meritorio — cuando se trataba de proteger los derechos de los emigrantes ante las autoridades estadounidenses- $y$, en otras, antidemocrático - cuando implicaba reconocer los derechos políticos de los que se van-. En suma, el análisis de estas conductas son determinantes para entender por qué la élite partidista determina si la emigración transnacional mexicana con voto extraterritorial es inocua, arriesgada o amenazante en términos electorales. A continuación, con base en la teoría de juegos - y tomando en cuenta la interacción de la Cámara baja (1994-2003) — ofreceremos una explicación.

\section{INTERACTIVIDAD MULTIPARTIDISTA E}

INCLUSIÓN DEL VOTO EXTRATERRITORIAL

El 1 de diciembre de 1994, el gobierno de Ernesto Zedillo inauguraba sus gestiones con un legado político conflictivo, como consecuencia del levantamiento del Ejército Zapatista de Liberación Nacional (EZLN) (Reforma, 3 de enero de 1994) registrado, exactamente, once meses antes. Estos hechos se consumaban justo en el momento en que México anunciaba su ingreso en la dinámica del primer mundo, con la entrada en vigor del Tratado de Libre Comercio de América del Norte (TLCAN); además de los asesinatos políticos del candidato del PRI a la presidencia de la República, Luis Donaldo Colosio (Reforma, 24 de marzo de 1994), y del secretario general del partido, José Francisco Ruíz Massieu (Reforma, 29 de septiembre de 1994). El panorama sombrío, para Ernesto Zedillo, se agudizó unos pocos días después de su toma de posesión, por un manejo inadecuado de las finanzas públicas —en específico, la balanza de pagos - que ocasionaría una de las crisis económicas más graves de la historia contemporánea de México; a este episodio se le conoce como «error de diciembre», o «efecto tequila» por sus repercusiones económicas a nivel mundial. 
En este contexto nacional e internacional, una de las principales acciones del nuevo gobierno fue buscar un acuerdo, a nivel del país, que incluyera a todos los partidos políticos con registro ante el Instituto Federal Electoral (IFE), organizaciones políticas sin registro y grupos que representaran los intereses de la sociedad civil, como los medios de comunicación y los sindicatos. El PRI-gobierno buscaba dar salida a la severa crisis política y financiera que atravesaba el país a través del diálogo, el consenso junto con la negociación, y no importaba que mantuviera la mayoría relativa en la Cámara de Diputados, con el 47.8\% de los escaños, y en el senado, con el $60.1 \%$. Es decir, las condiciones metaconstitucionales de gobierno unificado, alta disciplina y diarquía unificada. ${ }^{8}$ La prioridad del PRI-gobierno era la estabilidad del país y, para ello, necesitaba contar con la cooperación del resto de los organismos institucionales, esto permitió la interacción partidista y abrió la puerta a los ciudadanos mexicanos en el extranjero.

Si utilizamos el recurso analítico de la teoría de juegos para examinar la reforma electoral de 1996, tendremos elementos para entender el porqué de los resultados, una vez explicadas la interacción corroborada con datos empíricos. En seguida se presenta el ordenamiento de las preferencias y la explicación analítica del juego de estrategias dominantes asumidas por el PRI-gobierno y el bloque opositor, en las negociaciones sobre la reforma electoral de 1996.

TABLA 1

PREFERENCIAS PARTIDISTAS

\begin{tabular}{|c|c|c|c|c|c|}
\hline \multicolumn{2}{|c|}{ Preferencias PRI-gobierno } & \multicolumn{3}{c|}{ Preferencias bloque opositor } \\
\hline PRI & Oposición & Valor & PRI & Oposición & Valor \\
\hline r & r & 4 & R & R & 4 \\
\hline r & R & 3 & r & R & 3 \\
\hline R & r & 2 & R & r & 2 \\
\hline R & R & 1 & r & r & 1 \\
\hline
\end{tabular}

La primera preferencia del PRI-gobierno estaba a favor de una reforma que hiciera más equitativa la competencia electoral. Si bien, el PRi había ganado la Presidencia en 1994, sin impugnaciones por fraude electoral como en 1988, el entonces presidente, Ernesto Zedillo, reconocía la inequidad en el financiamiento

\footnotetext{
${ }^{8}$ Todo lo anterior se puede encuadrar en un modelo desarrollado por Nava, Weldon y Yañez (2000), que muestra cómo las tres condiciones metaconstitucionales (gobierno unificado, alta disciplina del PRI y diarquía unificada) permitían a los presidentes de la República surgidos del PRI sacar adelante, en el Congreso, las reformas que se proponían implementar.
} 
público a los partidos políticos opositores y en el acceso a los medios de comunicación, hasta ese momento claramente a favor del pRI. Por lo que se entiende que su primera preferencia fuera votar una reforma electoral que él mismo había promovido para maximizar sus utilidades, además, le beneficiaba que la oposición hiciera lo mismo, una vez otorgadas las suficientes concesiones que ésta exigía, entre las que incluía el voto extraterritorial. Así, el PRI-gobierno ganaba en legitimidad con la reforma, sobre todo después de los acontecimientos de violencia política de 1994, al tiempo que se presentaba a las elecciones de 1997 con reglas del juego que aún le favorecían, pero que se exhibían como imparciales para la competencia electoral democrática.

La segunda posición en el orden de preferencias, para el PRI-gobierno, fue aprobar la reforma electoral con o sin el apoyo del bloque opositor, toda vez que contaba con la mayoría relativa en la Cámara de Diputados y el control en la Cámara Senadores para su ratificación (condiciones metaconstitucionales). Además, en la lógica del PRI-gobierno, la reforma resultaba coherente con lo ofrecido por Zedillo, ya que consideraba que la mayor parte de las exigencias de la oposición habían sido consideradas e incluidas en el Acuerdo Político Nacional, antesala protocolaria de la reforma electoral firmada por todos los partidos políticos con representación en el Congreso y con el presidente como testigo. La tercera y la cuarta preferencias no tiene sentido considerarlas, dado que el PRI-gobierno fue el principal impulsor de la reforma electoral y sería irracional pensar que pudiera votar en su contra. Aunque en la realidad pudiera existir esa posibilidad, se considera muy remota debido a que el PRI-gobierno consideraba prioritario abrir canales de expresión institucional que desactivaran opciones violentas, como las del EZLN, a la vez que ahuyentaran las preocupaciones sobre inestabilidad que provocaron los asesinatos políticos y la crisis económica de 1994. De acuerdo con lo anterior, se puede apreciar que el ordenamiento de las preferencias del PRI-gobierno tenía una estrategia dominante con respecto a la reforma electoral de 1996: siempre fue $r$.

Respecto al bloque opositor, su posición frente a la reforma electoral la podemos analizar considerando varias circunstancias. Para éste, el compromiso alcanzado con el PRI-gobierno - y suscrito en el Acuerdo Político Nacional- le representaba un avance sustantivo acorde a sus intereses, al incluir la mayor parte de las exigencias que había solicitado, incluso, también, el voto en el extranjero. Sólo restaban dos puntos importantes donde el PRI-gobierno no cedería, ya que desde el principio había dejado en claro su negativa: las candidaturas de coalición y el descenso de los montos de financiamiento público a los partidos políticos, principalmente al PRI. Por otra parte, las exigencias de equidad y vigilancia del proceso electoral, que demandaba el bloque, se habían cumplido con la autonomía definitiva del IfE y con las atribuciones reconocidas al Tribunal Electoral del Poder Judicial de la Federación (TRIFE); además, se garantizaba el acceso a los medios comunicativos y la elección del jefe de gobierno del Distrito Federal, principalmente. 
Los cálculos realizados por el bloque opositor, sobre la reforma electoral, predecían saldos positivos que los ubicaban en una posición de avanzada respecto a procesos electorales anteriores. Aun así, la postura de la oposición fue rechazar la iniciativa del PRI-gobierno, porque creía que eso maximizaba sus beneficios frente a la opinión pública y a su militancia, de cara a las elecciones de 1997. En esta lógica se entiende que la primera preferencia, para el bloque, fuera que el PRI-gobierno desistiera de la iniciativa planteada y se aceptaran, por completo, las peticiones que exigía la oposición. La segunda estrategia fue dejar sólo al PRI-gobierno con su reforma, ya que le reportaba ganancias graduales pero sustantivas $y$, de antemano, contaba con información suficiente para pensar que, con su apoyo o sin él, el PRI-gobierno aprobaría la reforma al contar con las condiciones metaconstitucionales y porque el contexto, nacional e internacional, así lo demandaba. Al maximizar el bloque sus utilidades, la estrategia dominante fue negarse, rotundamente, a la reforma $R$, pero, al mismo tiempo, que fuera aprobada por el PRI. En resumidas cuentas: $R>r>C$. Donde la ruptura es preferida que la reforma y la reforma es preferida a la continuidad.

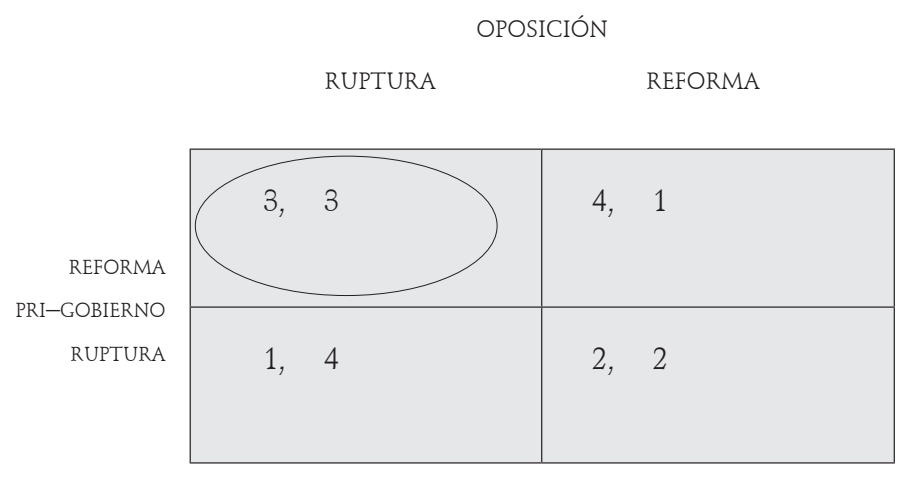

NOTA: EQUILIBRIO ÚNICO QUE SE PRESENTA EN LA CASILLA 3, 3.

Analizando las posiciones asumidas por los protagonistas, se trata de un juego de estrategias dominantes donde siempre, para el PRI-gobierno, su mejor respuesta a la estrategia del Bloque Opositor será r, mientras que la mejor réplica de la oposición, ante los planteamientos del PRI, será $R$. Con esto, se puede afirmar que existe un único equilibrio en la casilla superior izquierda de la matriz 3, 3, ya que ninguna otra opción mejoraría la posición de las partes. Los datos empíricos demuestran que no hubo acuerdo final, sobre la reforma electoral de 1996, entre el PRI-gobierno y el bloque opositor, pero, al mismo tiempo, los hechos nos demuestran que nunca se trató de un juego de suma-cero donde, todo lo que ganó una parte, lo perdió la otra.

Las interacciones entre los dos bandos arrojaron saldos positivos para ambos, lo que nos permite hablar de equilibrio eficiente en términos de elección social, ya que la nueva ley electoral garantizaba elecciones competidas y compe- 
titivas, respeto al voto de los ciudadanos y la certeza de contar con una autoridad electoral autónoma a la vez que competente, como lo era el IfE. Por el lado de los partidos políticos, el PRI aseguraba su supervivencia en un escenario cada vez más riguroso y hostil a su presencia y, en el caso del bloque opositor, se pertrechaba rumbo a los comicios de 1997 que, a la luz de los resultados electorales, le darían, por primera vez en la historia moderna de México, el control de la Cámara de Diputados y el Gobierno del Distrito Federal. El voto extraterritorial, a pesar de su aprobación, no se pudo reglamentar.

\section{ESTRATEGIAS E INTRANSIGENCIAS EN \\ LA FALLIDA REFORMA ELECTORAL}

Durante la interactividad partidista de la segunda legislatura, aún en el periodo presidencial de Zedillo (1997-2000), la situación nacional e internacional era muy diferente al escenario previo a la reforma de 1996. En primer lugar, el PRI-gobierno consideraba que ya existían las condiciones de equidad en el juego electoral y que, la mejor muestra de ello, eran los resultados de las elecciones de 1997. En segundo lugar, ahora lo importante era preparar la elección presidencial de 2000, en la que cada uno de los bandos políticos procuraba las mejores condiciones de competencia. En este contexto previo a la sucesión presidencial, se entiende que la estructura de las preferencias del PRI-gobierno y los partidos de oposición, agrupados en el llamado bloque opositor, fueran opuestas durante la interacción que se presentó en la fracasada reforma electoral de 1999.

TABLA 2

PREFERENCIAS

\begin{tabular}{|c|c|c|c|c|c|}
\hline \multicolumn{2}{|c|}{ Preferencias PRI-gobierno } & \multicolumn{3}{c|}{ Preferencias Oposición } \\
\hline PRI & Oposición & Valor & PRI & Oposición & Valor \\
\hline R & R & 4 & r & r & 4 \\
\hline r & r & 3 & R & r & 3 \\
\hline R & r & 2 & r & R & 2 \\
\hline r & R & 1 & R & R & 1 \\
\hline
\end{tabular}

NOTA: DONDE $R$ SIGNIFICA RUPTURA Y $r$ SIGNIFICA REFORMA

Podemos observar que la primera preferencia del PRI-gobierno fue, simplemente, que no hubiera reforma electoral y que el bloque opositor no presentara iniciativa al respecto, ya que sabía que las exigencias de la oposición estaban lejanas a un punto de acuerdo mínimo para sus intereses, una vez realizados sus cálculos y estrategias. Además, los incentivos para cooperar eran reducidos, 
después de analizados los efectos de la reforma de 1996 sobre los resultados de las elecciones de 1997, donde habían perdido la mayoría relativa en la Cámara de Diputados y la importante plaza del Distrito Federal. La segunda preferencia estaba en aprobar una reforma moderada o parcial, que diera respuesta a ciertas peticiones de la oposición, como reglamentar el voto por correo desde el extranjero para ciudadanos con credencial para votar, concesión que se otorgaba confiando en la escasa participación electoral de los mexicanos allende las fronteras, pero excluyendo lo relativo a las candidaturas de coalición y la rebaja al financiamiento público de los partidos. Se trataba que el coste de ampliar el electorado no fuera mayor a los saldos negativos de una postura intransigente ante la elección de 2000. Su tercera preferencia se situaba, de nuevo, en rechazar la reforma y que, en todo caso, la oposición la aprobara en la Cámara de Diputados, con la certeza de que, posteriormente, sería rechazada en el senado con mayoría priísta, la apuesta estaba en cargar con el coste del rechazo sabiendo que era mucho menor ante la posibilidad de aceptar las candidaturas de coalición, el tope de financiamiento y el voto en el extranjero. Como se puede observar, su última prioridad, siendo irracional, era aprobar la reforma y que el bloque opositor no lo hiciera, volviéndose a repetir lo ocurrido en 1996.

Por su parte, el bloque opositor tenía unas preferencias que, en apariencia, resultaban contradictorias pero, si se analizan con detalle, no lo eran en realidad. En primer lugar, la principal preferencia de la oposición era que se aprobara la reforma electoral sin necesidad de ceder en ninguna de sus peticiones, es decir, que el PRI-gobierno dijera sí a las candidaturas de coalición, descenso del financiamiento público y voto en el extranjero. Como segunda preferencia, estaba la aprobación de la ley en la Cámara de Diputados sin el consenso del PRI-gobierno, este supuesto se confirma en el entendido de que la oposición sabía que, si aprobaba la reforma, ésta sería rechazada, invariablemente, en la Cámara de Senadores. Entonces, la estrategia de no negociar y no ceder con el PRI-gobierno implicaba rechazar la propia ley: se perdía con el rechazo pero se ganaba presentando al PRI-gobierno como obstáculo principal al cambio político y, a ellos, por el contrario, como impulsores de la democracia. Además, la oposición fundamentaba sus creencias en la posibilidad de alcanzar la presidencia de la República con la ley electoral vigente, sin necesidad de correr riesgos con una nueva que incluyera el voto en el extranjero. No existía la certeza de que el voto emigrante les beneficiaría y sí, por el contrario, la inseguridad de que les podía perjudicar un manejo fraudulento desde el exterior. Asimismo, los resultados de 1997 alentaron, al bloque opositor, a creer en la posibilidad de una victoria con la ley vigente, con un IFE autónomo, un PRI-gobierno dividido y, sobre todo, con los recursos económicos que el Código Federal de Instituciones y Procedimiento Electorales (COFIPE), de 1996, les había otorgado. La tercera y última preferencia no se presenta debido a que resulta irracional que el bloque opositor votara contra una ley que ellos mismos habían propuesto. 


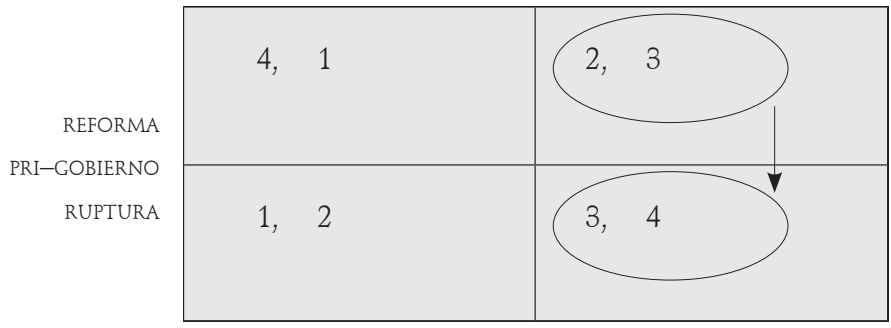

NOTA: EQUilibRIO DEFICIENTE EN LA CASILLA 2, 3, CON DILEMA DEL PRISIONERO AL NO EXISTIR COMUNICACIÓN QUE LOS LLEVARA A OTRO EQUILIBRIO EFICIENTE EN TÉRMINOS DE ELECCIÓN SOCIAL EN LA CASILLA 3, 4

La interacción que las posiciones partidistas nos presentan es la misma que ocurre con el dilema del prisionero. Para el PRI-gobierno, la mejor estrategia fue $R$ y, para el bloque opositor, $r$, con lo cual se presenta un equilibrio en la casilla superior derecha 2, 3 que, contrastada con los datos empíricos, fue al final la postura asumida, aunque ineficiente en términos de elección social. En este caso, la estrategia principal del PRI-gobierno fue rechazar la reforma, ya que los incentivos para cooperar con la nueva ley electoral, tal como la planteaba el bloque, implicaba correr el riesgo de perder la presidencia en 2000. Dicha creencia se fundamentaba en el retroceso electoral registrado en 1997. Por su parte, la estrategia dominante del bloque opositor fue $r$, porque si se aprobaba salía ganando, al incluir los tres puntos básicos que demandaba: candidaturas de coalición, tope al financiamiento público al PRI así como voto en el extranjero; y, por otro lado, si la iniciativa se venía abajo, el bloque confiaba en que la ley electoral vigente le podía dar el triunfo.

Pero el dilema del prisionero se presenta ya que, debido a la falta de información fiable sobre la posible orientación del voto emigrante, no existieron incentivos para que cambiaran su postura a otro equilibrio en el que los dos encontraran mejores pagos, por ejemplo, en la casilla posterior derecha 3, 4. De haber existido suficiente comunicación o aptitud, se pudo haber avanzado a una reforma electoral, así sea parcial, que permitiera votar a los mexicanos en el extranjero, es decir, eficiente en términos de elección social. El haber excluido el voto emigrante, en las elecciones presidenciales de 2000, es el resultado de la falta de cooperación entre el PRI-gobierno y el bloque opositor.

\section{INMOVILIDAD PARTIDISTA E INERCIA INSTITUCIONAL}

Durante la LVIII Legislatura (2000-2003), la interacción partidista era completamente distinta debido al triunfo electoral del panista Vicente Fox, luego de 
setenta años de hegemonía priísta. En este sentido, el Partido Acción Nacional (PAN), ahora PAN-gobierno, deseaba aprobar las principales reformas estructurales y económicas que, a su juicio, requería el país - principalmente la energética y fiscal - pero no tenía incentivos, en ese momento, de impulsar una nueva ley electoral. Para el PRI no existían estímulos y, por lo tanto, no quería reformas, ni las estructurales ni la política, su empeño estaba en bloquear todas las iniciativas del Ejecutivo. Por su parte, el Partido de la Revolución Democrática (PRD) tenía nulo interés en las reformas económicas, en cambio sí tenía motivos para la reforma política, pero no tenía prisa, ya que vislumbraba lejos el 2006 y, antes, había que considerar los resultados intermedios de 2003. En cambio, para los partidos pequeños (PVEM, PT y Convergencia, principalmente), la reforma se presentaba como el instrumento clave de supervivencia, sobre todo si lograban ampliar las prerrogativas económicas. A pesar de que existían fuertes incentivos en los partidos pequeños, no tiene caso ahondar en ellos pues, por sí solos, no tenían capacidad para reformar una ley, al menos que participaran dentro de una alianza mucho mayor, cosa que tampoco sucedió.

Como lo hicimos anteriormente, ahora presentaremos la estructura de las preferencias del PAN-gobierno y los partidos opositores, en atención a la interactividad que se presentó en la LVIII Legislatura, sin que se acordaran las reformas fiscal, energética y electoral.

TABLA 3

PREFERENCIAS

\begin{tabular}{|c|c|c|c|c|c|}
\hline \multicolumn{2}{|c|}{ Preferencias PAN-gobierno } & \multicolumn{3}{c|}{ Preferencias oposición } \\
\hline PAN & Oposición & Valor & PAN & Oposición & Valor \\
\hline r & r & 4 & R & R & 4 \\
\hline r & R & 3 & r & R & 3 \\
\hline R & r & 2 & R & r & 2 \\
\hline R & R & 1 & r & r & 1 \\
\hline
\end{tabular}

NOTA: DONDE $R$ SIGNIFICA RUPTURA Y $r$ SIGNIFICA REFORMA

Podemos observar que la primera preferencia del PAN-gobierno fue que las reformas estructurales fueran aprobadas por consenso entre todas las fuerzas políticas con representación en la Cámara baja. La segunda preferencia consistía en aprobar una serie de reformas estructurales parciales que le permitieran afrontar, con éxito, las elecciones intermedias de 2003, aunque aquellas no fueran por acuerdo de todos los partidos políticos pero, al menos, los suficientes para alcanzar la aprobación, con ello conseguía, de manera parcial, sus objetivos y dejaba, a sus principales adversarios políticos, la etiqueta de obstructores del cambio. La tercera prioridad consistía, al menos, en lograr una reforma estructural fragmen- 
taria, aprobada por los partidos de oposición sin el consentimiento del PAN-gobierno. Esta era una apuesta arriesgada pero, por lo menos, le brindaba algún margen de maniobra en la gestoría de la agenda económica: la ecuación era perder en la Cámara a cambio de ganar en el gobierno. La última posibilidad era el fracaso de las reformas y que ninguna de ellas se concretara de acuerdo a lo planeado por los estrategas de PAN-gobierno, vaticinio que, finalmente, se cumplió.

Por su parte, los partidos de oposición tenían, como prioridad principal, que ninguna reforma estructural y política fuera aprobada por el PAN-gobierno o por otros partidos opositores. Simplemente porque la estrategia era obstaculizar la gestión del gobierno, confiando en que las elecciones de 2003 castigarían al presidente Fox, por las promesas incumplidas. En segundo lugar, la preferencia consistía en rechazar cualquier reforma estructural donde el PAN-gobierno corriera con el riesgo de exhibir y aprobar una reforma parcial con escasa incidencia en la gestión económica, además que se viera afectado en las elecciones de 2003. La tercera opción consistía en apoyar una reforma sin que el PAN-gobierno lo hiciera, con el evidente riesgo de darle, al PAN-gobierno, beneficios en la gestión económica sin ser esa el propósito. Por último estaba la aprobación del paquete de reformas estructurales por consenso de todos los partidos en la Cámara, consecuentemente, esta posibilidad se presenta como irracional para los intereses electorales de los partidos de oposición.

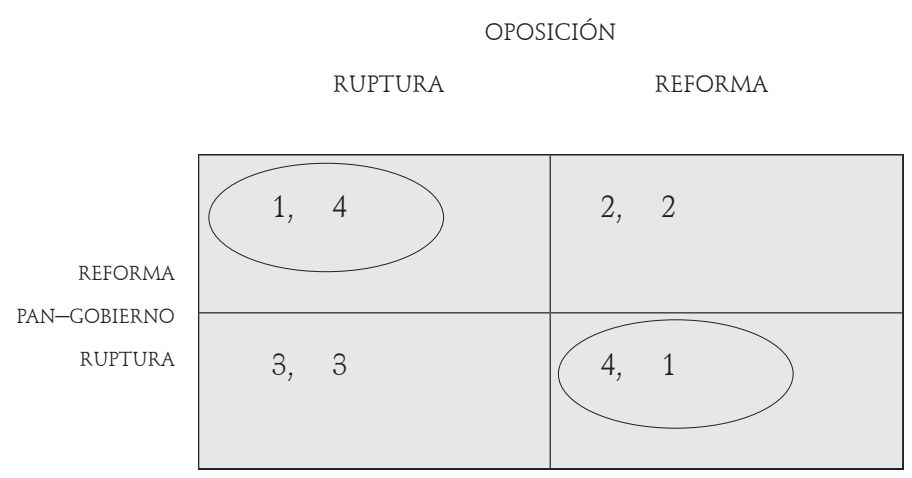

NOTA: EQUILIBRIO DEFICIENTE EN LA CASILLA 2, 3, CON DILEMA DEL PRISIONERO AL NO EXISTIR COMUNICACIÓN QUE LOS LLEVARA A OTRO EQUILIBRIO EFICIENTE EN TÉRMINOS DE ELECCIÓN SOCIAL EN LA CASILLA 3, 4.

Como podemos ver, la interacción partidista que se nos presenta es la misma que ocurre en el equilibrio de Nash. ${ }^{9}$ Para el PAN-gobierno, la mejor estrategia

\footnotetext{
${ }^{9}$ El equilibrio de Nash es cuando la estrategia de cada jugador es la mejor respuesta a la estrategia que juega el otro. La elección racional señala que en algunos contextos el equilibrio describe, de forma bastante fidedigna, el comportamiento real de los individuos.
} 
fue $r y$, para los partidos opositores, $R$, con lo cual se presenta un equilibrio en el que ningún actor político desea moverse de casilla porque no obtiene beneficios sin que otros lo pierdan. Como se observa en las casillas 1 y 4 , este resultado es un equilibrio ineficiente en términos de elección social. Lo anterior se comprueba si analizamos las estrategias dominantes de los actores: para el PAN-gobierno, la estrategia principal fue aprobar la reforma, ya que los incentivos para apoyar las reformas estructurales eran altos, sobre todo si se trataba de un elemento clave de la gestión económica de cara a las elecciones intermedias de 2003. Por su parte, la estrategia dominante de los partidos de oposición fue $R$, porque si se aprobaban las reformas se alejaba la pretensión de evidenciar la incapacidad del PAN-gobierno en gestionar la economía y, sobre todo, que lo anterior se reflejara en las elecciones intermedias, como un eslabón más de la cadena en busca de la presidencia en 2006.

El equilibrio ineficiente, en términos de elección social, queda de manifiesto por el simple hecho de que las reformas estructurales, que necesitaba el país, no fueron logradas. En este mismo escenario se vio envuelta la reforma electoral de segunda generación que reglamentaría el voto de los mexicanos en el extranjero. ¿̇A qué obedeció esta actitud partidista? Simplemente a que las reformas estructurales y el reconocimiento de la ciudadanía transnacional, para emigrantes mexicanos, dependen de los incentivos concretos que tenga la élite parlamentaria, una vez realizados los cálculos de costo-beneficio político y electoral que significaría apoyar una iniciativa de esta naturaleza.

\section{REFLEXIONES FINALES}

La pregunta central, de este artículo, podemos hacerla de la siguiente manera: ¿̇por qué fue imposible la reglamentación del voto extraterritorial para ciudadanos emigrantes mexicanos durante las legislaturas comprendidas entre 1994 y 2003? La respuesta la ofrece el análisis de la teoría de juegos: no existieron los incentivos suficientes en el PRI, PAN Y PRD para que el voto extraterritorial fuera reglamentado y, por el contrario, se impuso la incertidumbre sobre la orientación electoral de 10 millones de emigrantes mexicanos, en un sistema político caracterizado por elecciones competidas. Además, la duda institucional se vio acentuada porque la emigración transnacional mexicana tiene una larga historia de movilización política plural, por ello no resulta sencillo instrumentar estrategias de cooptación y clientelismo político para ningún partido.

La interacción partidista, analizada en este artículo, ofrece elementos de análisis suficientes para comprobar lo anterior: la reglamentación del voto extraterritorial es un asunto de votos antes que un debate normativo sobre la pertenencia. Los cambios institucionales en las reglas del juego político en México, en ese periodo (1994-2003), no fueron suficientes para incorporar a los ciudadanos 
residentes en el extranjero como parte de la comunidad política. Este trato, de las élites políticas y partidistas, a los emigrantes no es congruente con los principios democráticos básicos y de representación política; así lo demostraron en las negociaciones para la reforma de 1996, en el fallido intento en 1999 y en la inercia institucional de la rvir Legislatura. En dichas coyunturas dominó la versión utilitarista de la política antes que una versión comprensiva de la pertenencia al demos: خ̇a quién beneficiarían o perjudicarían esos 10 millones de votos potenciales?

Si examinamos, detenidamente, la realidad política del periodo comprendido en esas legislaturas podemos verificar lo que el análisis de este artículo plantea: las intenciones, por parte de los partidos, son de conveniencia electoral antes que ideológicas. En el Pan había distintas posturas respecto al voto, desde la indiferencia hasta el rechazo total. ${ }^{10}$ La primera tiene una lógica histórica ya que, previamente a 1997, el acercamiento del PAN con los emigrantes era nulo. La segunda etapa es consecuencia de los triunfos electorales en varios estados del país, sobre todo en aquellos con altos índices de emigración como Guanajuato, Jalisco, Aguascalientes, Baja California, Chihuahua; también por el perfil del emigrante de la década de los ochenta, más de clase media y con mayor escolaridad; pero sin duda, la razón principal del cambio, en la percepción del pan, fue descubrir las simpatías entre los ciudadanos emigrantes y esto se debe a Vicente Fox. ${ }^{11}$ El impasse final del PAN en 1999, al no aceptar la contrapropuesta del PRI-gobierno, se debió a que pensaba en la posibilidad de triunfar en las elecciones presidenciales sin voto extraterritorial. Lo ilógico para el PAN hubiera sido correr un riesgo innecesario de abrir el voto al extranjero, el resultado del 2 de julio de 2000 les dio la razón. Para la LVIII Legislatura, los escenarios eran distintos y, ya en el Ejecutivo, las estrategias fueron diversas.

Por su parte, el PRD siempre manifestó su apoyo al voto extraterritorial, aunque su postura tampoco fue homogénea. Hay dos razones principales: una normativa y otra más pragmática en lo político así como en lo electoral. La primera tiene una raíz histórica por el trabajo, junto con la convergencia ideológica entre los dirigentes de la «vieja» izquierda nacional y diversos grupos de trabajadores emigrantes mexicanos; sobre todo destacaba el activismo de Heberto Castillo al frente del Partido Mexicano de los Trabajadores (PMT) — después Partido Mexicano Socialista (PMS), finalmente convertido en $\mathrm{PRD}$ - así como la indiscutible figura carismática de Cuauhtémoc Cárdenas y su legado histórico entre los emigrantes

\footnotetext{
${ }^{10}$ Martínez Cossío señala que el diputado Castilla Peniche se quejaba: «[...] el PAN lo respaldó a través mía, dejaron que yo dijera todo lo que quisiera, pero realmente no vi una respuesta superafirmativa del partido. Yo siento que sí están a favor pero no lo ven como una prioridad [...]» (Martínez Cossío, 2001: 228).

${ }^{11}$ Esto fue notorio por el apoyo que recibió Vicente Fox a través del grupo Migrantes Mexicanos por el Cambio (Mimexca) (Martínez Cossío, 2000).
} 
mexicanos. ${ }^{12}$ La segunda fase obedece a un pragmatismo político y electoral, es decir, los cálculos del PRD les hicieron pensar que esta relación histórica, sumada a la inconformidad de los emigrantes con el gobierno, les garantizaba una suma de votos a su favor. La versatilidad de Porfirio Muñoz Ledo para incluir, en la reforma electoral de 1996, el voto en el extranjero obedece a ello, principalmente, y no tanto a creencias normativas sobre una ciudadanía transnacional. Otra cosa es que ese pragmatismo sea más ilusorio que cercano a la realidad, o bien, que el activismo del PRD hacia el emigrante sea una postura no sincera y más de apariencia, ya que en el fondo puede dominar la incertidumbre que ocasiona el desconocimiento de la orientación del voto extraterritorial, como parece que ocurrió en las negociaciones para la reforma política de 1999. Para el periodo 2000-2003, el PRD fue claro en su discurso, no así en su intencionalidad no explícita.

La postura más extrema, respecto al voto extraterritorial, es la del PRI, por ello se entiende que hayan promovido y legislado la Ley de No Pérdida de la Nacionalidad Mexicana en 1996 (doble nacionalidad), que daba respuesta a algunos de los reclamos de la emigración mexicana, pero que no incluía los derechos políticos. Si el PRI aceptó el voto extraterritorial, en la reforma electoral de 1996, lo hizo pensando en los otros puntos más importantes de la agenda política, bastante agudizada por la crisis político-económica y los mensajes que llegaban del ámbito internacional: cambio o desaparición. No es difícil adivinar las percepciones del PRI respecto al voto extraterritorial, si se analiza la maltrecha relación de los gobiernos priístas con la emigración mexicana, misma que les hacía suponer que los votos de los emigrantes serían en su contra, ${ }^{13}$ eso por un lado y, por el otro, el discurso nacionalista del PRI que siempre ha encontrado, en Estados Unidos, la causa de todos los males del país. El uso de la retórica para presentar, a los emigrantes mexicanos, como objetos animados desde el poder estadounidense, por sí solo era una razón más que suficiente para negar el voto extraterritorial, ese fue el discurso utilizado durante todo el proceso de reformas políticas en la década de los noventa y, aún en la actualidad, persiste en algunos reconocidos priístas como Diego Valadés o Jorge Carpizo.

Finalmente -y para concluir - podemos celebrar que, el 30 de junio de 2005, la interacción partidista con representación en el Congreso haya decido

${ }^{12}$ Dice Martínez Cossío que el PRD tiene una relación más intensa con los emigrantes que cualquier otro partido político, ya que cuenta con una vinculación institucional en su esquema organizativo encargada de los asuntos de los militantes en Estados Unidos y, además, porque el socialismo mexicano (parte del PRD) siempre ha apoyado el voto extraterritorial (Martínez Cossío, 2000).

${ }^{13}$ A partir de la presidencia de Carlos Salinas de Gortari se ha podido observar un intento de restablecer la relación del PRI con la emigración mexicana; por ejemplo, se implementó el Programa Paisano, el Programa de Solidaridad Internacional entre Mexicanos, el Programa para las Comunidades Mexicanas en el Extranjero, entre otros. Sin embargo, estas políticas transnacionales del PRI -gobierno siempre consideraron estrechar los vínculos culturales, sociales y económicos, pero nunca los políticos. 
reglamentar el voto de los mexicanos en el extranjero. La decisión de la LXI Legislatura, sin duda, se debe a que los cálculos partidistas arrojan más ganancias que costes y a que la incertidumbre electoral se encuadra en los márgenes de control partidista. De esa manera se explica que la modalidad del voto postal se haya impuesto a la petición de la mayoría de los emigrantes: que el proceso electoral se realizará a través de la instalación de casillas en embajadas y consulados mexicanos. El utilitarismo partidista refleja esta medida adoptada por diputados y senadores mexicanos. La explicación es muy simple: de todas las alternativas posibles para instrumentar el sufragio extraterritorial, los senadores y diputados escogieron la peor de ellas, el sufragio por correo.

El voto postal es el método que implica mayores probabilidades de manipulación, tanto en el origen como en la recepción de los votos emitidos, más aún, es un mecanismo que no existe en los procesos electorales que se realizan dentro del territorio nacional. Si se piensa en potenciar la participación electoral, que no es el caso, sin duda se escogió el instrumento inadecuado. No será sencillo, para un emigrante mexicano, solicitar con antelación la papeleta electoral y posteriormente mandarla, sobre todo si no existe un precedente a nivel nacional que dé confianza y certeza. Por otra parte, existe el riesgo del clientelismo y compra de sufragios que, en el caso del voto postal, se acentúa aún más, como ocurrió, en fechas recientes, con las elecciones parlamentarias de Galicia así como la permanente sospecha de compra de votos emigrantes españoles en Argentina, Venezuela y Cuba. Por el contrario, la opción del voto en las embajadas y consulados garantiza mejores resultados en términos de limpieza electoral, como ocurre, exitosamente, con Colombia o Perú, que realizan elecciones ejemplares en el extranjero.

Otro de los aspectos negativos del proceso, sino el más importante, se refiere a la idea de que el voto en el extranjero fue adoptado como una política de discriminación positiva. Cuando se legisla una ley de esta naturaleza, siempre habrá otro segmento de la población que se vea afectado por la medida. Es decir, si en un principio los emigrantes se sentían ciudadanos de segunda, ahora habrá mexicanos, residentes en México, que se perciban agraviados y sean ellos los que experimenten dicha condición. Si bien no tendrían razón, están en su derecho de manifestarlo. Lo cierto es que este hecho es imputable, nuevamente, al Congreso, porque tal medida debió ser acompañada de una política de reconocimiento de la emigración transnacional mexicana, proceso que implicaba, de manera necesaria, que las demandas de los ausentes fueran admitidas por los no emigrantes. Desafortunadamente, la deliberación pública no estuvo presente cuando esto ocurrió, como en muchos otros asuntos de la política mexicana.

Sin duda será muy interesante, en un futuro, analizar la interacción de los partidos que hicieron posible el voto extraterritorial. Entonces, la teoría de juegos seguirá siendo una herramienta útil para analizar esa política, donde lo racional está en las expectativas y no en los resultados, para desgracia de muchos actores políticos implicados. 
BIBLIOGRAFÍA

Acuña, Rodolfo (1986), América Ocupada, México, Editorial Era.

Becerra Ramirez, Manuel (2000), "Nationality in Mexico», en T. Alexander Aleinikoff y Douglas Klusmeyer (eds.), From Migrants to Citizens. Membership in a Changing World, Washington, Carnigie Endowment for Internacional.

Becerra, Ricardo, Pedro Salazar y José Woldenberg (2000), La mecánica del cambio político en México: elecciones, partidos y reformas, México, Cal y Arena.

(1996), La reforma electoral de 1996, México, Fondo de Cultura Económica.

Bustamante, Jorge A. y Wayne A. Cornelius (coords.) (1989), Flujos migratorios mexicanos hacia Estados Unidos, México, Fondo de Cultura Económica.

Bustamante, Jorge (1983), Espaldas mojadas: materia prima para la expansión del capital norteamericano, México, El Colegio de México.

Bustamante, Jorge, Daniel Delaunay y Jorge Santibáñez (1997), Taller de medición de la migración internacional, Tijuana, Colegio de la Frontera Norte, Institut Francais de Recherche Scientifique Pour le Développement e Coopération.

Calderón, Leticia (1997), «Vivir a dos tiempos. Actitudes políticas de inmigrantes mexicanos», tesis de doctorado en Ciencias Sociales, Sede México, Facultad Latino-Americana de Ciencias Sociales.

(coord.) (2003), Votar en la distancia. La extensión de los derechos políticos a migrantes, experiencias comparadas, México, Instituto Mora.

Calderón, Leticia y Jesús Martínez (2002), La dimensión política de la migración mexicana, México, Instituto Mora.

Carpizo, Jorge (1991), El presidencialismo mexicano, México, Siglo XXI.

Castillo, Pedro G. y Antonio Ríos (1989), México en Los Ángeles, México, Alianza Editorial Mexicana.

Cosío Villegas, Daniel (1975), La sucesión presidencial, México, Joaquín Mortiz.

Dresser, Dense (1995), "Consecuencias transfronterizas de la política mexicana», en Abraham F. Lowenthal y Katrina Burgess (comps.), La conexión México-California, México, Siglo XXI.

Duverger, Maurice (1986), Los partidos políticos, Fondo de Cultura Económica.

ELIzondo, Carlos y Benito Nacif (comps.) (2002), Lecturas sobre el cambio político en México, México, CIDE-Fondo de Cultura Económica.

Fitzgerald, David (2000), Negotiating Extra-Territorial Citizenship. The Mexican Migration and the Transnational Politics of Community, Monograph 2, San Diego, University of California.

Gamio, Manuel (1930), Quantitative estimate sources and distribution of Mexican inmigration into the United Status, México, Talleres Gráficos Editorial-Diario Oficial.

Gómez-Quiñones, Juan (1973), Sembradores, Ricardo Flores Magón y el Partido Liberal México, Los Ángeles, Aztlán Publications. 
GonzÁlez Gutiérrez, Carlos (1995), "La diáspora mexicana en California», en Abraham F. Lowenthal y Katrina Burgess (comps.), La conexión México-California, México, Siglo Xxi.

Griswold del Castillo, Richard (1979), The Los Angeles Barrio 1850-1890: A Social History, Berkeley, The California University Press.

Gutiérrez, David (1995), Walls and Mirrors. Mexican American, Mexican Immigrants and the Politics of Ethnicity, Berkeley, University of California Press.

MarTínez Cossío, Nayamín (2000), «żLa incorporación del México de afuera? Un análisis sobre el voto de los mexicanos en el exterior», tesis de maestría en Sociología Política, México, Instituto Mora.

(2001), «El voto extraterritorial: el desencuentro partidista frente a la extensión de la ciudadanía», en Arturo Santamaría Gómez et al., Mexicanos en Estados Unidos: la nación, la política y el voto sin fronteras, Culiacán, Partido de la Revolución Democrática, Universidad Autónoma de Sinaloa.

MARTínez Saldaña, Jesús (1993), «At the Periphery of Democracy: The Binational Politics of Mexican Immigrants in Silicon Valley», tesis de doctorado en Antropología Social, California, Universidad de Berkeley.

Meyer, Lorenzo y Josefina Zoraida Vázquez (1994), México frente a Estados Unidos. Un ensayo histórico, México, Fondo de Cultura Económica.

Moctezuma Longoria, Miguel (2000), "Clubes de migrantes, expresión organizada del migrante colectivo», en Lázaro Cárdenas Batel y Gonzalo Badillo Moreno (coords.), Los derechos de los migrantes mexicanos en Estados Unidos, México, Cámara de Diputados lvir Legislatura, Fundación para la Democracia.

Montejano, David (1991), Anglos y mexicanos en la formación de Texas, 1936-1986, México, Alianza Editorial.

NAvA, María del Carmen, Jeffrey Weldon y Jorge Yánez (2000), "Cambio político, presidencialismo y producción legislativa en la Cámara de Diputados», en Germán Pérez y Antonia Martínez (comps.), La Cámara de Diputados en México, México, flacso, Cámara de Diputados, Editorial Porrúa.

Parra Barbosa, José Francisco (2000), «Cambios en la participación política de los mexicano-americanos en California: la fuerza del voto en los noventa», tesis de maestría en Estudios México-Estados Unidos, EneP Campus Acatlán, UNAM.

Pérez, Germán y Antonia Martínez (comps.) (2000), La Cámara de Diputados en México, FlACSO-Cámara de Diputados-Editorial Porrúa, México.

Portes, Alejandro (1996), "Global Villagers. The Rise of Transnational Communities», en The American Prospect, vol. 7, no. 25.

PorTes, Alejandro, Luis Guarnizo y Patricia Landolt (1999), "The study of transnationalism: pitfalls and promise of an emergent research field», en Ethnic and Racial Studies, vol. 22, no. 22.

(1999), "Conclusion: Towards a new world-the origins and effects of transnational activities», en Ethnic and Racial Studies, vol. 22, no. 22. 
PrZeWorski, Adam (1987), «Marxismo y elección racional», en Zona Abierta, no. 45. (1988), Capitalismo y socialdemocracia, Madrid, Alianza Editorial.

(1995), Democracia y mercado: reformas políticas y económicas en la Europa del Este y América Latina, Cambridge, Cambridge University Press.

RaAT, W. Dirk (1993), Los revoltosos. Rebeldes mexicanos en los Estados Unidos. 19031923, México, Fondo de Cultura Económica.

Santamaría Gómez, Arturo (1994), La política entre México y Aztlán, relaciones chicano-mexicanas del 68 a Chiapas 94, México, Universidad Autónoma de Sinaloa, California State University.

(2000), "La doble nacionalidad y la doble ciudadanía», en Lázaro Cárdenas Batel y Gonzalo Badillo Moreno (coords.), Los derechos políticos de los mexicanos en Estados Unidos.

SAntamaría et al. (2001), Mexicanos en Estados Unidos: la nación, la política y el voto sin fronteras, Culiacán, Partido de la Revolución Democrática y Universidad Autónoma de Sinaloa.

SiLVA-Herzog Márquez, Jesús (1999), El antiguo régimen y la transición en México, México, Planeta, Joaquín Mortiz.

Szekely, Gabriel (2003), "El voto de los mexicanos en el extranjero», memoria del Coloquio Internacional El Voto de los Mexicanos en el Extranjero, Toluca, Instituto Estatal Electoral.

Weber, Devra, Roberto Melvilla y Juan Vicente Palermo (2002), El inmigrante mexicano: la historia de su vida: entrevistas completas, Manuel Gamio, México, SEGOB, Instituto Nacional de Migración, The Regents of the University of California, CIESAS.

Woldenberg, José (2002), La construcción de la democracia, México, Plaza y Janés.

\section{PERIÓDICOS}

Reforma, "Chocan Ejército y guerrilla», 3 de enero de 1994.

Reforma, "Asesinan a Colosio», 24 de marzo de 1994.

Reforma, "Asesinan a Ruíz Massieu», 29 de septiembre de 1994.

La Jornada, "Adiós al PRI», 3 de julio de 2000.

Reforma, «Fox tira al PRI», 3 de julio de 2000.

El Universal, «Debacle priísta, nadie domina el Congreso», 3 de julio de 2000.

La Jornada, "Adiós al PRI», 3 de julio de 2000.

Reforma, «Fox tira al PRI», 3 de julio de 2000. 